\title{
Ein neues Fragment von Rudolfs von Ems ,Barlaam und Josaphat' in der Stiftsbibliothek Göttweig
}

\author{
Astrid Breith
}

$\int l$ m Zuge des Projekts Manuscripta Mediaevalia Gottwicensia ${ }^{1}$ wurde im März 2018 in der Bibliotheca minor des Benediktinerstifts Göttweig (Niederösterreich) ein beschnittenes Doppelblatt eines bislang unbekannten Fragments von Rudolf von Ems ,Barlaam und Josaphat ${ }^{6}$ entdeckt. Es fungiert als Einband eines Druckes aus dem Jahr 1589, es kann aber auch erst in späterer Zeit hierfür verwendet worden sein. Der Trägerband hat den Titel Tractatus de censuris ecclesiasticis, cum appendice de usuris \& Cambiis, omnibus praesertim Clericis perutilis of necessarius, authore Doctore Cattaneo, Ord: Praedicat: Illustrissimi Archipraesulis, ac Principis Salisburgensis Theologo. [...] Pataviae MDLXXXIX [Passau 1589] und wird in Göttweig unter der Signatur Fragm. germ. 3 [= Bibliotheca minor, LVII.L.2.3, Einband] geführt. ${ }^{2}$

Das Fragment misst noch $170 \times 210 \mathrm{~mm}$, es wurde querformatig aufgeklebt und zeigt drei Spalten mit einer Spaltenbreite zwischen 6o und $70 \mathrm{~mm}$, der erschlossene Schriftraum von zwei Spalten beträgt mindestens $\mathrm{I} 60 \times \mathrm{I} 20 \mathrm{~mm}$. Als vorhandene Maße einer Seite liegen somit noch $\mathrm{I} 70 \times \mathrm{I} 40 \mathrm{~mm}$ vor, es darf aber wohl von einem ursprünglichen Seitenmaß von mindestens $190 \times$ I6o $\mathrm{mm}$ ausgegangen werden. Zwischen Spalte eins und zwei sind Löcher einer ehemaligen Heftung erkennbar, so dass die ursprüngliche Anlage der Handschrift als zweispaltig angenommen werden kann. Jede Spalte umfasst mindestens 27 Zeilen, wobei der untere Rand des Fragments jeweils mit dem Abschluss der Spalte einhergeht. Am oberen Rand wurde das Pergamentblatt um die Kante gebogen, auf den jeweiligen Spiegeln lässt sich noch zusätzlich ein Vers erkennen. Die Verse sind abgesetzt gehalten, jeder erste Vers ist ausgerückt und beginnt mit einem Großbuchstaben, wobei diese nicht durch rote Striche hervorgehoben sind. Diese Art der Texteinrichtung mit ausgerückten Anversen bildet ein Merkmal, das im I3. und I4. Jahrhundert überwiegend aus Handschriften des ostbairischen, österreichischen und böhmischen Raums bekannt ist. ${ }^{3}$ Rote zweizeilige Lombarden markieren im vorliegenden Fragment jeweils einen Abschnittsanfang. Da das Ende von Spalte 2 (Vers I40,5) und der Anfang von Spalte 3 (Vers I40,6) unmittelbar aufeinander folgen, muss die ursprüngliche Handschrift mit einer Zeilenzahl von 27-28 ausgeführt worden sein. Die exakte Angabe der Zeilenzahl muss trotz des Anschlusses der Verse I40, 5 f. offen gehalten werden, da aufgrund der divergierenden Versangaben der Ausgaben von Köpke und Pfeiffer etwaige unter dem aufgeklebten Vorsatzblatt unsichtbare Plusverse nicht ausgeschlossen werden können. ${ }^{4}$

Eine Suche im ,Handschriftencensus' ergab für die Kriterien ,26-28 Zeilen' und ,Verse abgesetzt' nur drei Treffer: Die Handschrift Freiburg i. Br., UB, Hs. 480 (Sigle D) (https://handschriftencensus.de/I0699) ist zwar nicht vollständig, umfasst aber doch 136 Blätter und weicht in der Einrichtung vom Göttweiger Fragment ab - die Verse sind dort gleichmäßig untereinander gesetzt und $\mathrm{ru}^{-}$ briziert, es gibt keine eingerückten Abverse. Zum zweiten Treffer der Abfrage, London, BL, MS Add. I0288, Bl. I2-I9, 4I-88, I2I-152 (Fragment 2, https://handschriftencensus.de/I097) liegen derzeit keine Abbildungen vor, aus der 
Beschreibung von Robert Priebsch ${ }^{5}$ geht jedoch hervor, dass auch hier die Anfangsbuchstaben rubriziert sind, zudem scheint die Gestaltung der Initialen von jener im Göttweiger Fragment abzuweichen. Das gleiche Ausschlusskriterium trifft auch zu auf Berlin, SBB-PK, Fragm. 93b (Fragment Io, https://handschriftencensus.de/I738), ohne Abbildung, auch dieses Fragment weist keine eingerückten Abverse auf. ${ }^{6}$

Das vorliegende Fragment ist in einer Textualis vom Ende des 13. / Anfang des 14. Jahrhunderts geschrieben und weist Merkmale der bairischen Schreibsprache auf. Es ist nicht identisch mit dem verschollenen Göttweiger Barlaam-Fragment Nr. I4 (https://handschriftencensus.de/Io6o) und wird $\mathrm{ab}$ sofort unter den im Handschriftencensus angeführten Überlieferungszeugen als Fragment mit der Nummer 39 geführt. ${ }^{7}$

Identifizierung und Textabgleich des Fragments erfolgten nach der Ausgabe von Friedrich Karl Köpke (I8I8). Herangezogen wurde zudem die Ausgabe von Franz Pfeiffer (I843), dessen Verszählung von der Köpkes manchmal abweicht. In der Transkription wurde die Schreibung des Fragments übernommen. Hierbei erwies sich eine textliche Abweichung als auffällig: In Vers I4O,I7 führen beide Ausgaben das Adjektiv libtez/liebtez an, das neue Göttweiger Fragment nennt hier cleinez (s.u. und Anm. 9). Die im Editionsbericht gemeldete kritische Edition des Werkes könnte Licht in die Überlieferungszusammenhänge dieses Werkes bringen, von dem - den Neufund eingerechnet inzwischen 52 Textzeugen bekannt sind. ${ }^{8}$

\section{Transkription des Fragments}

Spalte I, Anfangsbuchstaben fehlen:

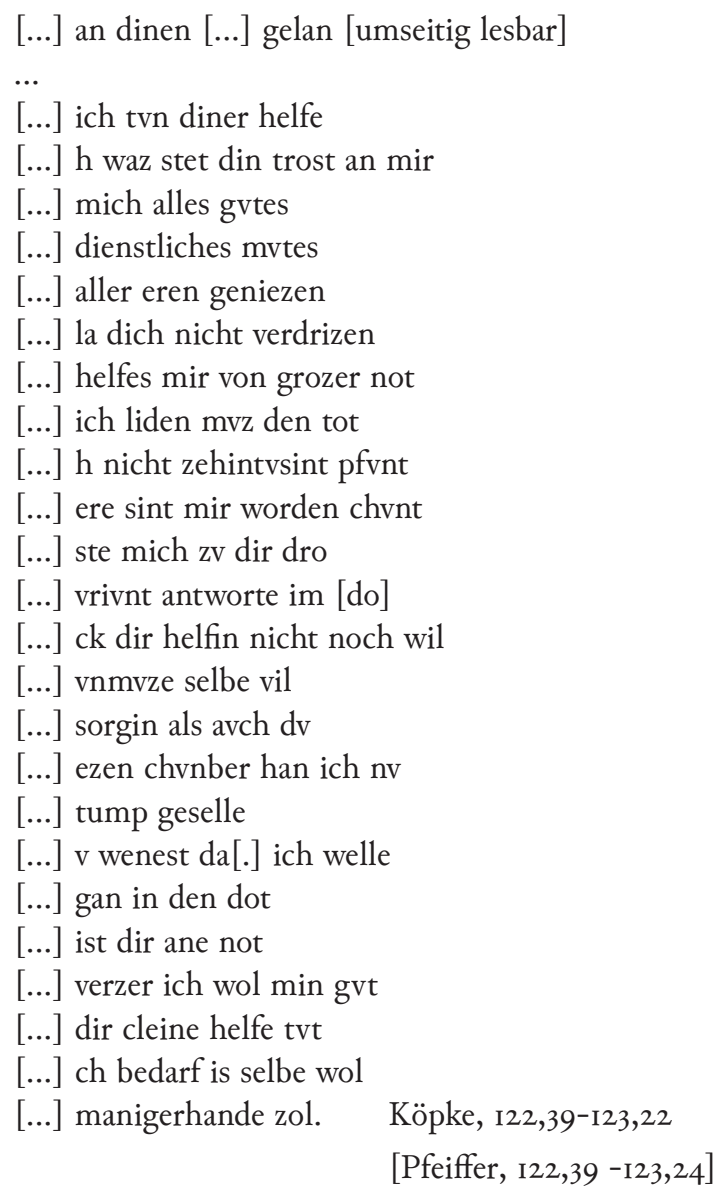


Spalte 2, großenteils unlesbar:

Mit $[\ldots]$ chin

vo[...] waz

vil [...] do daz

Do ditz [...] bewart vnd $[. .$.$] gespart$

Im wa $[\ldots]$ versait daz er [...] heit

Dem her[...] sagen ditz $[\ldots]$ beg $[\ldots]$

Daz er $[. .$.$] lan$ der ch[...] solden gan

Dvrch [...] stat ob [...] phat

Nv si irg [...] sich beg $[\ldots]$ do

Ein liec[...] liechten dvrch [...] elin

Do si wv[...] si hu [...] ar

Der chv[...] man da daz [...] bran

Des nam [...] der do sahen $[\ldots .$.

In der er [...] Köpke, $139,20-140,5$

[Pfeiffer, I39,22-I40,7]
Spalte 3:

[...] mit vuge wol [umseitig lesbar]

Dem iz zv hvse tochte dicz was ein gvt armer man

Der truc die besten cleider an di der chunich ie gesach

Als er da mit volge iach

Hie vor dem selben loche saz sin wip ir dinest niht vergaz Sie bracht im ein cleinez ${ }^{9}$ glas dar inne im bereitet was

Sin trinchen daz trvc sie im hin als erz genam sie stvnt vor im

Mit vreudin trat sie vnde spran [...] si sanch den svezisten sanc

Vil vroliche vnd schone gar in dem besten done

den ir deheiner ie vernam der dar mit dem chvnige quam si lopte ir man mit vrevdin $\mathrm{g}[. .$.

Den chvnich alda nicht verdroz ern nemes vlizeclichen war

Si wunderte algeliche gar daz disen lvoten was bereit

Von armvt di groste armicheit die in da vor ie wart bechant

Köpke, I40,6-I40,3I [Pfeiffer, I40,8-I40,33]

\section{Kontakt}

Astrid Breith

Österreichische Akademie der Wissenschaften · Institut für Mittelalterforschung · Hollandstr. II-I3 · IO2O Wien E-Mail: astrid.breith@oeaw.ac.at

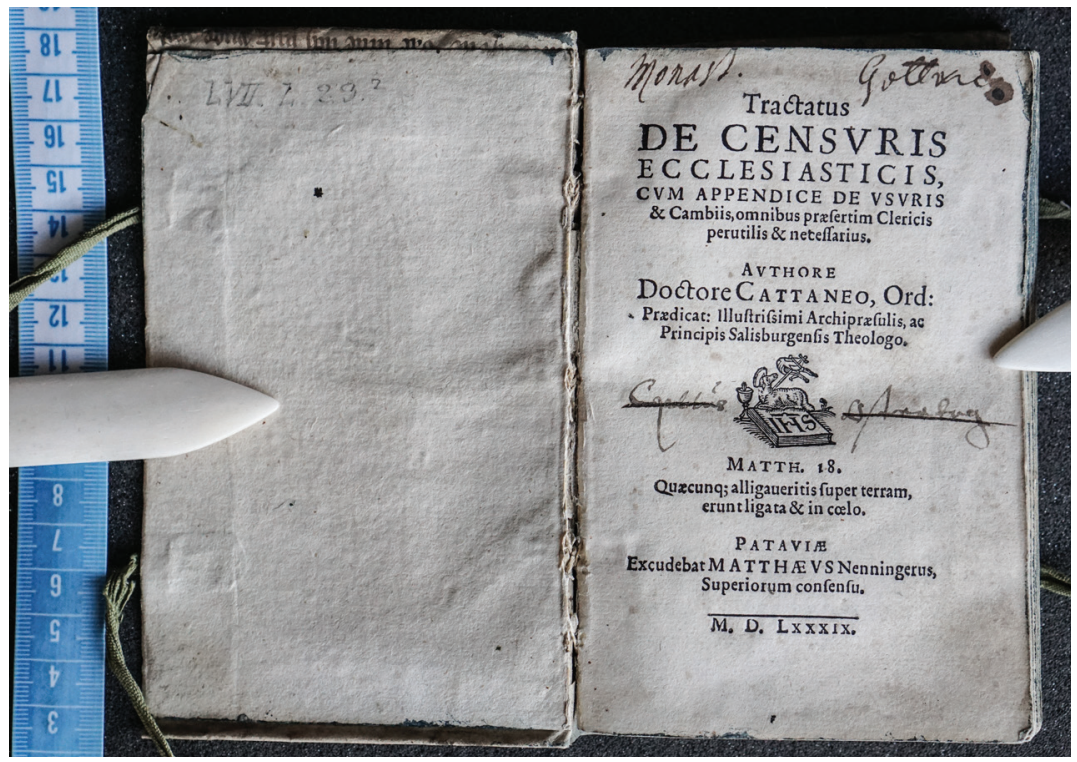

Abb. I: Göttweig, Bibliotheca minor, LVII.L.2.3, Trägerband mit einem Pergamentfragment, jetzt Göttweig, Fragm. germ. 3, als Einband (Foto: M. Lichtenwagner 202I) 


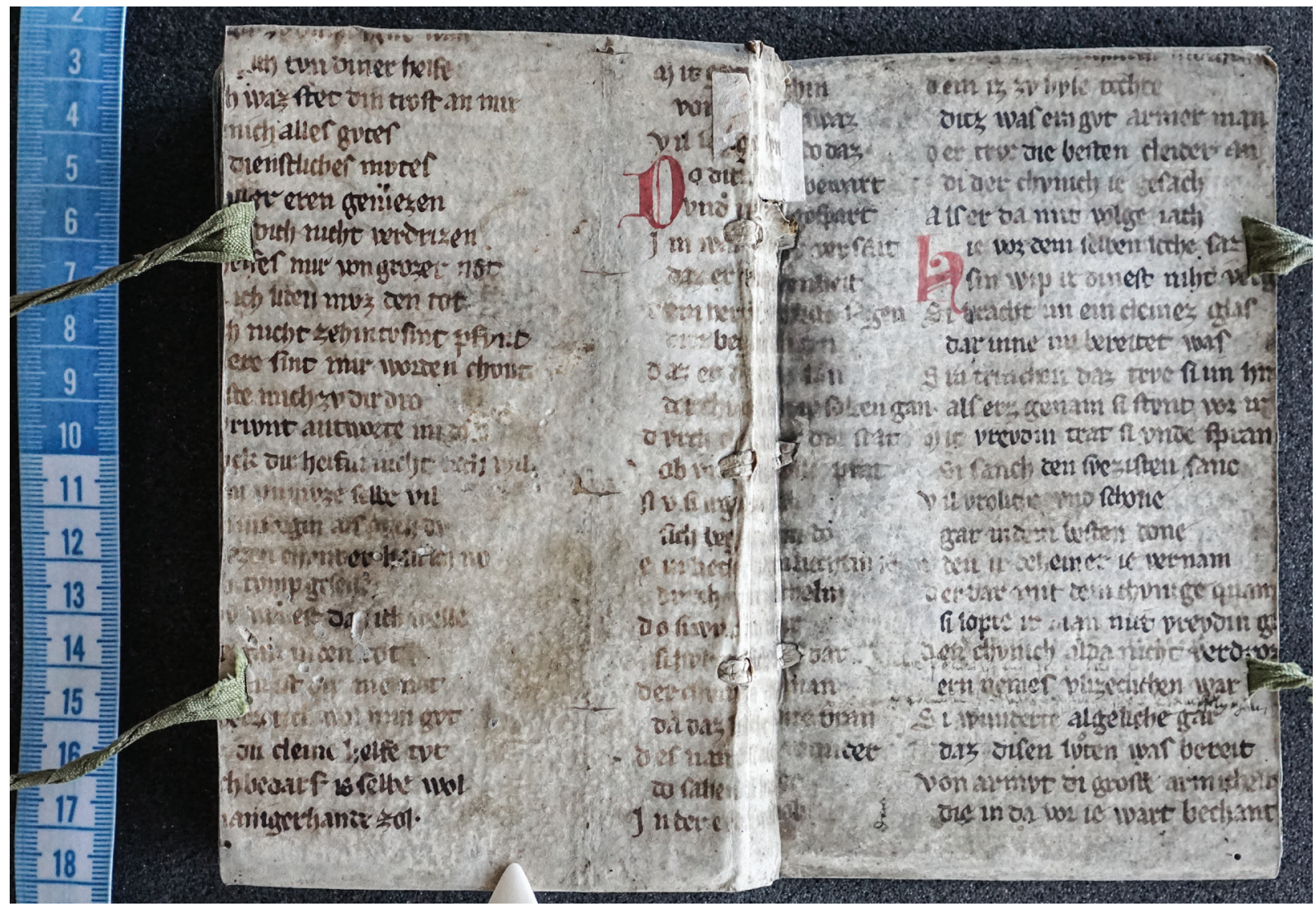

Abb. 2: Das geöffnete und gewendete Buch zeigt die Anlage als zweispaltige Handschrift und die Einrichtung der Verse (Foto: M. Lichtenwagner 202I)

\section{Anmerkungen}

I Die Abteilung Schrift- und Buchwesen des Mittelalters des Instituts für Mittelalterforschung der Österreichischen Akademie der Wissenschaften erforscht seit 2013 den mittelalterlichen Buchbestand des Benediktinerstiftes Göttweig (Niederösterreich). Ein erstes vom Österreichischen Wissenschaftsfonds FWF gefördertes Erschließungsprojekt befasste sich mit den Handschriften des I2., I3. und I4. Jahrhunderts, seit 2020 wird in einem zweiten Projekt der Bestand des I5. Jahrhunderts in den Blick genommen <https://www.oeaw.ac.at/imafo/forschung/schrift-buchwesen/manuscripta-mediaevalia-austriaca/goettweig-benediktiner-stiftsbibliothek/> (I6.3.202I).

2 Siehe VDı6 C 1737, dort geführt unter dem Verfassernamen Sebastiano Cattaneo.

3 Karin Schneider, Paläographie und Handschriftenkunde für Germanisten. Eine Einführung (Sammlung kurzer Grammatiken germanischer Dialekte B. Ergänzungsreihe Nr. 8), Tübingen 1999, S. 133.

4 Friedrich Karl Köpke (Hg.), Barlaam und Josaphat von Rudolf von Montfort, Königsberg I8ı8 (nach Thorn / Toruń, Universitätsbibl., Rps 40/IV); Franz Pfeiffer (Hg.), Rudolf von Ems, Barlaam und Josaphat (Dichtungen des deutschen Mittelalters 3), Leipzig I843 (Nachdruck mit einem Anhang, einem Nachwort und einem Register von Heinz Rupp, Berlin 1965). Beide Ausgaben sind online aufrufbar über die Literaturdatenbank des ,Handschriftencensus oder direkt beim Werkeintrag: <http://www.handschriftencensus.de/werke/32I> (II.5.202I). - Köpkes Ausgabe von I8I8 liegen drei Handschriften zugrunde, nach denen er seinen Text einrichtete: $\mathrm{K}=$ Warschau, NB, Cod. $8097 \mathrm{III}<$ https://handschriftencensus.de/4064>, Kb = Torún, UB, Rps 40/IV $<$ https://handschriftencensus.de/4066> sowie Ke = Berlin SBB-PK, mgf $20<$ https://handschriftencensus.de/4377>. Pfeiffer übernahm Köpkes Text und ergänzte ihn, im Apparat seiner Ausgabe von I843 führt er darüber hinaus Lesarten aus fünf weiteren Handschriften und sieben Fragmenten an: A = Karlsruhe, BLB, Cod. Donaueschingen 73 $<$ https://handschriftencensus.de/IO55>, B = Straßburg, SB, Cod. B I44, verbrannt <https://handschriftencensus.de/58II>, 
C = München, BSB, Cgm I6 <https://handschriftencensus.de/I3II>, D = Freiburg i. Br., UB, Hs 480 $<$ https://handschriftencensus.de/I099> und E = München, BSB, Cgm $273<$ https://handschriftencensus.de/6orI>, zu den Fragmenten a-g, siehe Pfeiffer, I843, S. 407f.

5 Robert Priebsch, Deutsche Handschriften in England, Bd. 2, Erlangen I90I, S. I04f. (Nr. I20): „Blaue und rote, mit Arabesken verzierte Initialen, rot durchstrichene, herausgerückte grosse Anfangsbuchstaben der Verse [...]“.

6 Herzlicher Dank geht an dieser Stelle an die ,Handschriftencensus'-Redaktion in Marburg, die der Autorin eine unveröffentlichte Abbildung des Fragments zu Recherchezwecken zukommen ließ.

7 ,Handschriftencensus'-Eintrag zu dem neuen Fragment: <https://handschriftencensus.de/25962>. Siehe auch den Eintrag auf manuscripta.at: <https://manuscripta.at/?ID=46396>.

8 Eine von der DFG geförderte Neuedition des Textes nach Leithandschriftenprinzip mit Variantenapparat, Übersetzung und Kommentar wird von Mathias Herweg (Karlsruher Institut für Technologie) vorgenommen.

9 Vers I40,I7: Köpke [libtez; Pfeiffer [liebtez, jeweils ohne Kommentar im Apparat. 